\title{
Safety evaluation for railway vehicles using an improved indirect measurement method of wheel-rail forces
}

\author{
Jing Zeng ${ }^{1} \cdot$ Lai Wei $^{1} \cdot$ Pingbo $\mathrm{Wu}^{1}$
}

Received: 16 November 2015/Revised: 15 April 2016/Accepted: 18 April 2016/Published online: 6 May 2016

(C) The Author(s) 2016. This article is published with open access at Springerlink.com

\begin{abstract}
The wheel-rail force measurement is of great importance to the condition monitoring and safety evaluation of railway vehicles. In this paper, an improved indirect method for wheel-rail force measurement is proposed to evaluate the running safety of railway vehicles. In this method, the equilibrium equations of a suspended wheelset are derived and the wheel-rail forces are then be obtained from measured suspension and inertia forces. This indirect method avoids structural modifications to the wheelset and is applicable to the long-term operation of railway vehicles. As the wheel-rail lateral forces at two sides of the wheelset are difficult to separate, a new derailment criterion by combined use of wheelset derailment coefficient and wheel unloading ratio is proposed. To illustrate its effectiveness, the indirect method is applied to safety evaluation of railway vehicles in different scenarios, such as the cross wind safety of a high-speed train and the safety of a metro vehicle with hunting motions. Then, the feasibility of using this method to identify wheel-rail forces for low-floor light rail vehicles with resilient wheels is discussed. The values identified by this method is compared with that by Simpack simulation for the same low-floor vehicle, which shows a good coincidence between them in the time domain of the wheelset lateral force and the wheel-rail vertical force. In addition, use of the method to determine the high-frequency wheel-rail interaction forces reveals that it is possible to identify the high-frequency wheel-rail forces through the accelerations on the axle box.
\end{abstract}

\section{Jing Zeng \\ zeng@home.swjtu.edu.cn}

1 State Key Laboratory of Traction Power, Southwest Jiaotong University, Chengdu 610031, China
Keywords Wheel-rail force $\cdot$ Safety evaluation $\cdot$ Indirect method - Union safety domain - Wheelset derailment coefficient $\cdot$ Hunting motions $\cdot$ Cross wind $\cdot$ Low-floor vehicle

\section{Introduction}

Nowadays, a great deal of attention to railway industry is focused on the condition monitoring of railway vehicles. The importance of this issue is highlighted by occurrences of problems caused by poor vehicle dynamic conditions or operational environment. For instance, the hunting motions of railway vehicles caused by the wear of wheel and rail profiles may lead to safety problems; a high-speed train subjected to strong cross winds has the potential risk of overturning. For a new opened railway line, the track inspection train is commonly used to evaluate the quality of the tracks. For a new designed vehicle, however, the derailment safety and running behavior must be evaluated by on-track tests. When using dynamic tests to measure the dynamic indices of a track inspection vehicle and a new designed vehicle, we need instrumented wheelset and some accelerometers installed on the tested vehicles. However, the instrumented wheelset is known as an expensive device and involves a complicated calibration technology [1-7]. In addition, some structural modifications to the wheelset, e.g., drilling holes on the wheel hub, are necessary for the signal transmission and power supply, which means that this device is not suitable for long-term commercial operation of the train. Therefore, it has been a great concern to find some alternatives to the traditional method for wheelrail force measurement.

The inverse identification method is a new approach to determine the wheel-rail interaction forces. In the Chinese 
Standard GB5599-85 [8], the derailment safety of railway vehicles are evaluated by measurement of the bogie frame force and the wheelset derailment coefficient. In this method, strain gages are attached on the bogie frame and a calibration test needs to be conducted to obtain the relationship between the forces and structure deformations. In literatures $[9,10]$, inverse dynamic models combined with force identification methods were commonly used to measure the forces on other structures as the direct measurement requires either expensive device or complicated instrumentation. The methods were also applied to the railway industry. For instance, Xia et al. [11, 12] developed an inverse wagon model to estimate the wheel-rail interaction forces by measuring only the wagon body responses as inputs. In their work, the vehicle system was modeled with rigid bodies and the partial modal matrix method was adopted to predict the input loads. Uhl [13] put forward an inverse method for the identification of contact forces, using accelerometers on the axle box and the transfer function between response points and wheel-rail contact points. Zhu et al. [14] established an inverse dynamic model to identify the dynamic forces in railway vehicles, in which the validation tests were conducted on a full-scale roller rig. Mehrpouya et al. [15] used a finite element model for a railway freight vehicle to identify the forces applied to the wheelset. Tanaka et al. [16] proposed a method for estimating the extraordinary values of wheel load and lateral force using the easily measured axle box accelerations. Gao et al. [17] and Wang et al. [18] utilized specially calibrated force measuring devices to obtain the forces exerted on the axle box spring and the swing arm.

However, the inverse methods for determining the wheel-rail interaction forces described above are usually derived from vehicle system models. Consequently, these methods cannot avoid the complexity of wheel-rail creep force and normal contact force and are not applicable to nonlinear vehicle systems. To identify the nonlinear wheel-rail contact forces, solutions are obtained by combination of instrumented wheelset technology and advanced sensors in Japan. That is, Matsumoto et al. [19] developed a new method to measure the wheel-rail forces without sticking the strain gage or installing the slip ring on the wheelset. In their work, the lateral wheel-rail force was obtained from the wheel deformation by several non-contact gap sensors, while the wheel-rail vertical and longitudinal forces were obtained from the deformation of axle box spring and the strain of swing arm.

An indirect method for wheel-rail force measurement has been proposed by the authors in Ref. [20]. In the previous work, we compared the forces applied to the instrumented wheelset with those obtained using the indirect method. This indirect method was validated on the curved track in the loop test line, from which it was concluded that the identified forces had good coincidence in the time domain with the instrumented wheelset [21]. It was also validated by a high-speed passenger vehicle subjected to cross winds, which also showed that the identified results agreed well with that from instrumented wheelset [22]. In general, the relative error of the peak values was lower than $15 \%$.

In this paper, an improved indirect method for wheelrail force measurement is applied to evaluate the running safety of railway vehicles. The wheelset is treated as a rigid body, which means that although it is possible to measure the total lateral force of the wheelset, it is impossible to separate the lateral forces between the two wheels. To deal with this problem, a derailment safety domain by a combination of the wheelset derailment coefficient and the wheel unloading ratio is developed based on the classic Nadal's derailment criterion. For demonstration of its effectiveness, this indirect method are applied to the safety evaluation of railway vehicles in different scenarios, for instance, the cross wind safety of a high-speed train, and the safety of a metro vehicle with hunting instability. In addition, the feasibility of applying this method to identify the wheel-rail forces for low-floor light rail vehicles with resilient wheels, as well as highfrequency wheel-rail interaction forces, is also investigated.

\section{Derailment evaluation by indirect method for wheel-rail forces measurement}

The mechanism of the indirect method for wheel-rail force measurement is to establish the force and moment equilibrium equations by analyzing the forces exerted on the wheelset, as shown in Fig. 1. The unknown wheelset lateral force and wheel-rail contact forces can then be calculated from the inertial forces and suspension forces, which are obtained by measuring the accelerations on the axle box and the relative displacements of the primary suspension. For the vehicle with primary suspension, the wheelset or wheel axle lateral force, i.e., the so-called $H$ force, is given in Ref. [20] as follows:

$H=-m_{\mathrm{w}} a_{y}-F_{\mathrm{s} 1}-F_{\mathrm{s} 2}$,

where $m_{\mathrm{w}}$ is the wheelset mass; $a_{y}$ is the lateral acceleration of the axle box; $F_{\mathrm{s} 1}$ and $F_{\mathrm{s} 2}$ are the lateral forces of the left and right primary suspensions.

Ignoring the shift of wheel-rail contact points and the roll acceleration of wheelset, the wheel-rail vertical contact force sat the two sides of the wheelset, i.e., $Q_{\mathrm{L}}$ and $Q_{\mathrm{R}}$, are calculated as 


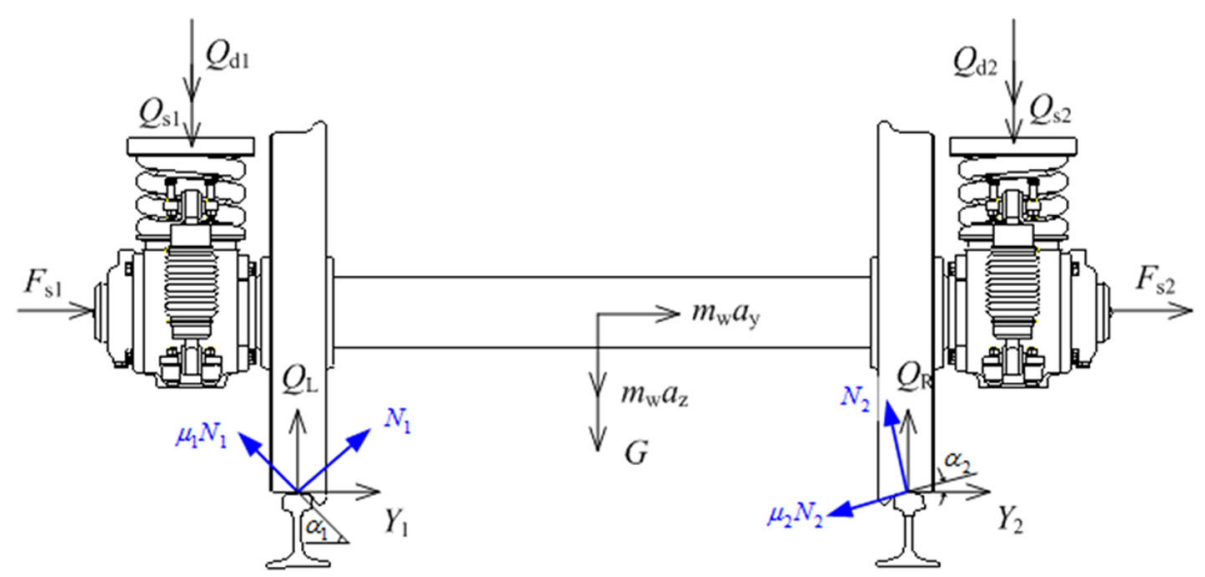

Fig. 1 Analysis of forces exerted on a suspended wheelset

$$
\begin{aligned}
Q_{\mathrm{L}} & =Q_{\mathrm{s} 1} \cdot\left(l_{\mathrm{s}} / 2+l_{\mathrm{c}} / 2\right) / l_{\mathrm{c}}+Q_{\mathrm{d} 1} \cdot\left(l_{\mathrm{d}} / 2+l_{\mathrm{c}} / 2\right) / l_{\mathrm{c}} \\
& -Q_{\mathrm{s} 2} \cdot\left(l_{\mathrm{s}} / 2-l_{\mathrm{c}} / 2\right) / l_{\mathrm{c}}-Q_{\mathrm{d} 2} \cdot\left(l_{\mathrm{d}} / 2-l_{\mathrm{c}} / 2\right) / l_{\mathrm{c}} \\
& +G / 2+m_{\mathrm{w}} a_{z} / 2+H \cdot r_{0} / l_{\mathrm{c}}
\end{aligned}
$$

and

$$
\begin{aligned}
Q_{\mathrm{R}} & =Q_{\mathrm{s} 2} \cdot\left(l_{\mathrm{s}} / 2+l_{\mathrm{c}} / 2\right) / l_{\mathrm{c}}+Q_{\mathrm{d} 2} \cdot\left(l_{\mathrm{d}} / 2+l_{\mathrm{c}} / 2\right) / l_{\mathrm{c}} \\
& -Q_{s 1} \cdot\left(l_{\mathrm{s}} / 2-l_{\mathrm{c}} / 2\right) / l_{\mathrm{c}}-Q_{\mathrm{d} 1} \cdot\left(l_{\mathrm{d}} / 2-l_{\mathrm{c}} / 2\right) / l_{\mathrm{c}}, \\
& +G / 2+m_{\mathrm{w}} a_{\mathrm{z}} / 2-H \cdot r_{0} / l_{\mathrm{c}},
\end{aligned}
$$

where $Q_{\mathrm{s} 1}$ and $Q_{\mathrm{s} 2}$ are the vertical forces of the primary springs; $Q_{\mathrm{d} 1}$ and $Q_{\mathrm{d} 2}$ are the vertical forces of the primary dampers; $a_{z}$ is the vertical acceleration of the axle box; $l_{\mathrm{c}}$ is the lateral distance between the wheel nominal running cycles; $l_{\mathrm{s}}$ is the lateral distance between the primary springs; $l_{\mathrm{d}}$ is the lateral distance between the primary vertical dampers; $G$ is the gravitational force of the wheelset; and, $r_{0}$ is the wheel radius.

The commonly used wheel derailment coefficient $Y / Q$ is defined as the ratio of the wheel-rail lateral force to the wheel-rail vertical force. According to the critical force state of wheel climbing derailment (see Fig. 1), the singlewheel derailment coefficient at the left and right wheel-rail contact point can be derived [23]:

$\frac{Y_{\mathrm{L}}}{Q_{\mathrm{L}}}=\frac{\tan \delta_{\mathrm{L}}-\mu_{\mathrm{L}}}{1+\mu_{\mathrm{L}} \tan \delta_{\mathrm{L}}}$,

$\frac{Y_{\mathrm{R}}}{Q_{\mathrm{R}}}=\frac{\tan \delta_{\mathrm{R}}+\mu_{\mathrm{R}}}{1-\mu_{\mathrm{R}} \tan \delta_{\mathrm{R}}}$,

where $Y_{\mathrm{L}}$ and $Y_{\mathrm{R}}$ indicate the left and right wheel-rail lateral forces, respectively; $Q_{\mathrm{L}}$ and $Q_{\mathrm{R}}$ are the wheel-rail vertical forces, respectively; $\tan \delta_{\mathrm{L}}$ and $\tan \delta_{\mathrm{R}}$ are the left and right wheel-rail contact angles, respectively; and $\mu_{\mathrm{L}}$ and $\mu_{\mathrm{R}}$ are the wheel-rail friction coefficients on the left and right sides, respectively.
If the left wheel-rail contact angle reaches the maximum flange angle, then the left wheel is in the critical condition of the flange climbing derailment. The roll angle of the wheelset is relatively small compared with the contact angle in the flange wheel. The wheel-rail vertical contact force applied at the left and right contact points can be expressed as

$Q_{i}=Q\left(1 \mp \frac{\Delta Q}{Q}\right)(i=\mathrm{L}, \mathrm{R})$,

where $Q$ is the nominal wheel-rail vertical force, $\Delta Q$ is the wheel unloading force, and the ratio of the wheel unloading force to the nominal wheel-rail vertical force is defined as the wheel unloading ratio $\Delta Q / Q$. The negative sign in Eq. (6) is related to the left flange climbing wheel and the positive sign to the right non-flanging wheel. In addition, the $H$ force can be written as

$H=Y_{\mathrm{L}}-Y_{\mathrm{R}}$.

Based on Eqs. (6) and (7), the wheelset derailment coefficient can then be written as

$\frac{H}{Q}=\frac{Y_{\mathrm{L}}}{Q_{\mathrm{L}}} \cdot\left(1-\frac{\Delta Q}{Q}\right)-\frac{Y_{\mathrm{R}}}{Q_{\mathrm{R}}} \cdot\left(1+\frac{\Delta Q}{Q}\right)$,

where the wheelset derailment coefficient is defined as the ratio of the $H$ force to the nominal wheel-rail vertical force.

In this indirect method, however, the wheelset is treated as a rigid body, which means that the left and right wheelrail lateral forces cannot be separated. To overcome this disadvantage, a derailment evaluation based on the wheelset derailment coefficient and the wheel unloading ratio is derived in this section. By substituting Eqs. (4) and (5) into Eq. (8), we obtain the derailment criterion based on the wheelset derailment coefficient as below: 


$$
\begin{aligned}
\frac{H}{Q} & +\left[\frac{\tan \delta_{\mathrm{L}}-\mu_{\mathrm{L}}}{1+\mu_{\mathrm{L}} \tan \delta_{\mathrm{L}}}+\frac{\tan \delta_{\mathrm{R}}+\mu_{\mathrm{R}}}{1-\mu_{\mathrm{R}} \tan \delta_{\mathrm{R}}}\right] \cdot \frac{\Delta Q}{Q} \\
& =\frac{\tan \delta_{\mathrm{L}}-\mu_{\mathrm{L}}}{1+\mu_{\mathrm{L}} \tan \delta_{\mathrm{L}}}-\frac{\tan \delta_{\mathrm{R}}+\mu_{\mathrm{R}}}{1-\mu_{\mathrm{R}} \tan \delta_{\mathrm{R}}} .
\end{aligned}
$$

Given the other wheel-rail contact parameters in Eq. (9), the wheelset derailment coefficient and wheel unloading ratio can be determined by the indirect method for wheel-rail force measurement. Based on the critical state of derailment, the boundaries of the safety domain can be determined, see Fig. 2. The black oblique line of the safety domain is determined from Eq. (9) with the contact angle of $70^{\circ}$ and the friction coefficient of 0.3. If the flange climbing wheel is loaded with the wheel unloading ratio of -1.0 , then no-flanging wheel is lifted from the rail. Finally, a triangular safety domain against derailment is determined. The vehicle can be assessed to be safe if the wheelset derailment coefficient and wheel unloading ratio are located in the left area of the black oblique line. It is seen that the safety area of the unloading flange wheel is smaller than that of the loaded wheel, which means that the flange climbing wheel possesses a higher derailment risk if the wheel is unloaded.

Besides, the wheel unloading ratio is only applicable to the situation that the wheel axle lateral force is zero or very small and should be used together with the derailment coefficient. The derailment evaluation by wheelset derailment coefficient $H / Q$ is similar to the wheel axle force limit which is applied to evaluate the wheel-rail interactions rather than the flange climbing.

Furthermore, the ' $H$ force criteria' in the Standard GB5599-85 can be derived by substituting Eq. (6) into Eq. (9):

$\frac{H+0.24 \cdot Q_{\mathrm{R}}}{Q_{\mathrm{L}}} \leq 1.0$.

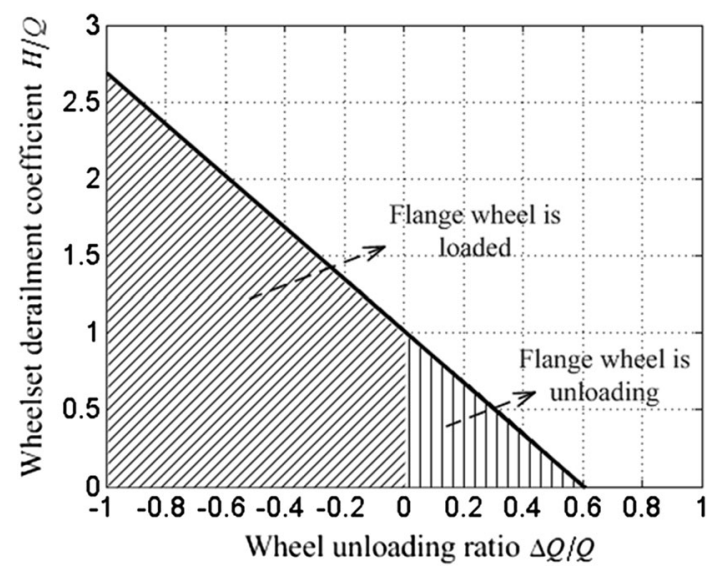

Fig. 2 Union safety domain based on wheelset derailment coefficient and wheel unloading ratio
In general, the new derailment criterion is equivalent to the traditional flange climbing derailment evaluation method and can be transfer to an equivalent form to the traditional method.

\section{Applications}

\subsection{Safety evaluation of high-speed train under cross winds}

Aiming at the high-speed train subjected to cross winds, a dynamic test using indirect method for wheel-rail force measurement was conducted in the Lanzhou-Xinjiang high-speed railway line. The environmental wind speed in the so-called 'hundred-mile wind area' between Urumqi and Hami was as high as $33 \mathrm{~m} / \mathrm{s}$. The axle load of the tested vehicle was $13.4 \mathrm{t}$ and the normal speed of the vehicle was $250 \mathrm{~km} / \mathrm{h}$. Accelerometers, laser transducers, and instrumented wheelset were installed on the test vehicle, as shown in Fig. 3. The goal of this test was to identify the $H$ force and the wheel vertical forces $Q_{i}$ using this indirect method, by measuring the accelerations on the axle box and the displacements of the primary suspension. Comparisons of the identified forces with the measured results from instrumented wheelset were introduced in Ref [22], which showed that the identified wheel vertical forces had perfect correlation with the measured results from the instrumented wheelset.

The running safety of the high-speed train under cross winds is affected by many factors, e.g., the running speed, wind characteristic, car body shape, etc. The system response and wheel-rail forces of the vehicle subjected to strong cross winds are quite complicated. Figure 4 gives the measured wheel-rail interaction forces in some particular locations using this indirect method. The vertical forces show that the wheel facing to the wind (windward side) is loaded while the wheel away from the wind (leeward side) unloaded. Meanwhile, the $H$ force synchronously increases with the wheel-rail vertical forces. The combination of the increasing lateral force and decreasing vertical force could result in a derailment risk.

For the traditional safety domain using the single-wheel derailment coefficient and wheel unloading ratio, the safety domain is presented as the rectangle type. The scatter diagram is plotted using the absolute values of $Y / Q$ and $\Delta Q / Q$. For the new-derived derailment criterion, the safety domain using the wheelset derailment coefficient and wheel unloading ratio is presented as the triangular type. If the flange climbing wheel is unloaded (positive), the wheel is at high risk of derailment. Conversely, the flange climbing wheel is relatively safe if the wheel is loaded (negative). The safety assessment based on the proposed 


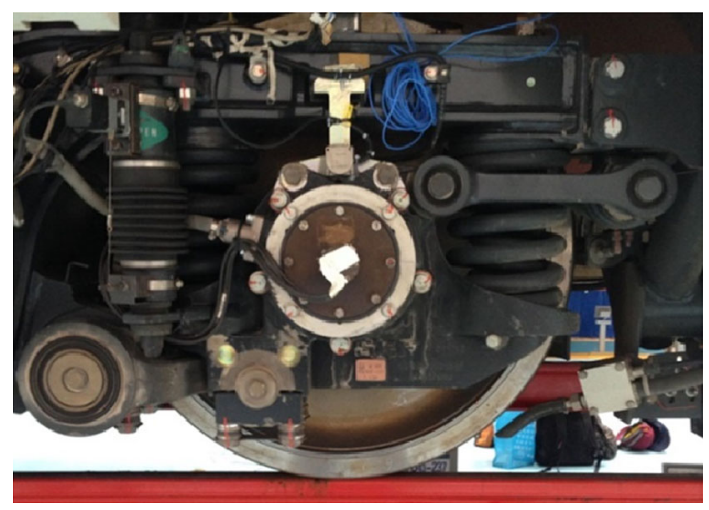

Fig. 3 Illustration of primary structures and test instruments of the tested high-speed vehicle

criterion is shown in Fig. 5. We can see that the safety indices of the head car subjected to cross winds that exceed the allowable limit in some locations, while the measured indices for the tail car under cross winds are within the safety limit.

\subsection{Safety evaluation of metro vehicle with hunting motions}

The indirect method for wheel-rail force measurement was also used to conduct a dynamic test for a metro vehicle. The axle load of the tested vehicle was $8.57 \mathrm{t}$ and the normal speed of the vehicle was nearly $100 \mathrm{~km} / \mathrm{h}$. In some locations of the track, the wheel-rail contact conicity of the worn wheel is very high, which may probably cause hunting motions of the vehicle system. The test instruments including accelerometers and laser transducers are shown in Fig. 6. The goal of this test was to identify the $H$ force and the wheel vertical forces $Q_{i}$ using this indirect method, by measuring the accelerations on the axle box and the displacements of the primary suspension. The safety evaluation of the metro vehicle with hunting motions is then carried out.

The running safety of the metro vehicle with hunting motions is affected by many factors, such as the running speed, wheel-rail contact relations, and track excitations. Figure 7 gives the measured wheel-rail interaction forces based on this indirect method. We can see that a very large $H$ force appears in some certain locations of the track and the waveform behaves as harmonic vibration, indicating that hunting instability occurs to the vehicle.

The scatter diagram for the safety assessment against derailment of the metro vehicle is shown in Fig. 8. For the derived derailment criterion above, the safety domain using wheelset derailment coefficient and wheel unloading ratio is presented as the triangular type. We can see that the safety indices of the metro vehicle under unstable conditions exceed the allowable limit, while that under stable conditions is within the safety limit. Thus, the occurrence of hunting motions to the metro vehicle has great influence on the derailment safety. Further measures, e.g., wheel re-profiling to reduce the contact conicity, should be taken to solve this problem.

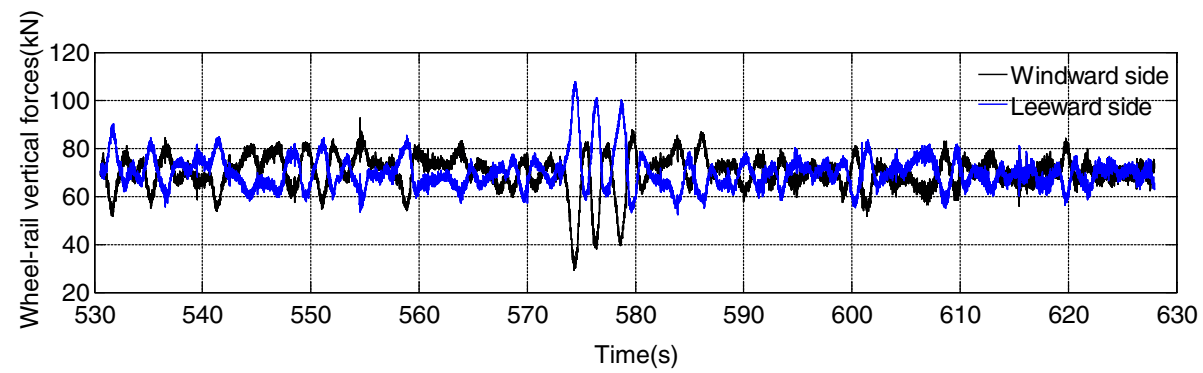

(a)

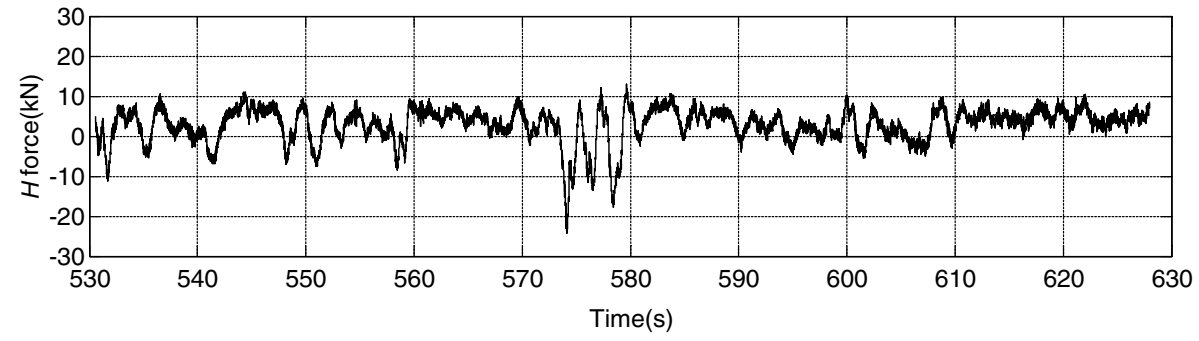

(b)

Fig. 4 Measured wheel-rail interaction forces of high-speed vehicle under cross winds. a Wheel-rail vertical forces. b $H$ force 


\subsection{Safety evaluation of low-floor vehicle with resilient wheels}

The traditional instrumented wheelset is not applicable to low-floor light rail vehicles (LRVs) with resilient wheels because the deformation of the resilient wheel is influenced by the rubber pad. An alternative method is to adopt the indirect method for wheel-rail force measurement. The forces exerted on the resilient wheelset are shown in Fig. 9. The idea of the indirect method for wheel-rail force measurement is also to establish force and moment equilibrium equations by analyzing the forces exerted on the resilient wheelset. The unknown forces $H$ and $Q_{i}$ can then be obtained from inertial forces and suspension forces, which can be determined by measuring the accelerations on the axle box and the relative displacements of the primary suspension. Besides, the interaction forces of the rubber ( $Y_{\mathrm{ei}}$ and $\left.Q_{\mathrm{ei}}\right)$ are difficult to measure because of the rotating contact position. The elastic rubber is herein treated as the primary suspension while the force is taken as the internal force. In principle, the methodology for the wheel-rail force measurement based on Eqs. (1), (2) and (3) is still applicable.

In this section, the simulation package Simpack is used to develop the low-floor vehicle model in which nonlinear wheel-rail kinematic constraints, nonlinear wheel-rail creep forces, and nonlinear suspensions are taken into account. The accelerations on the axle box, the displacements of the primary suspension, and the wheel-rail forces are generated from this model. To verify the indirect method theoretically, the wheel-rail forces determined from Eqs. (1), (2) and (3) are compared with the forces calculated by Simpack. The running speed for simulations is $55 \mathrm{~km} / \mathrm{h}$ on the curved track with a radius of $300 \mathrm{~m}$, and the measured track irregularities are adopted in the simulations. As can be seen from Fig. 10, this method can

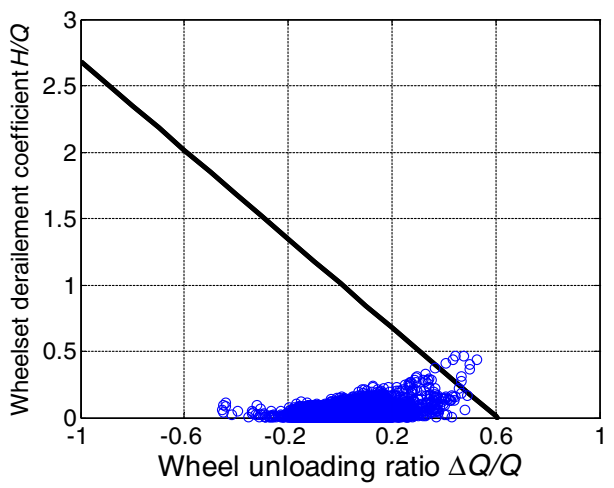

(a) provide good results for the $H$ force and the wheel-rail vertical forces $Q_{i}$, and is suitable for application. The small difference in the vertical force is caused by neglect of the rolling motion of the wheelset as well as the shift of wheelrail contact points. The good agreement between the identified and actual wheel-rail forces implies that the output data can be accurately obtained from the simulations. However, further feasibility tests on LRVs are necessary for the validation of the indirect method, since the actual running environments are more complicated than those considered in the simulations.

Actually, the simulated results can not reflect completely the real responses as there are a lot of undesired signals in practice. Unfortunately, for low-floor vehicles with small-radium resilient wheelset, there is no instrumented wheelset available, so the indirect method cannot be verified experimentally by measurements on an actual instrumented wheelset. Alternatively, the indirect method can be verified theoretically using Simpack.

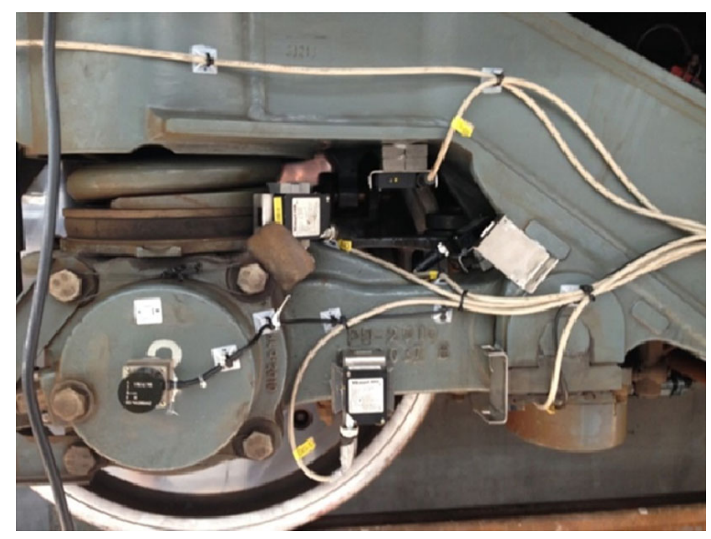

Fig. 6 Illustration of primary structures and test instruments of the tested metro vehicle

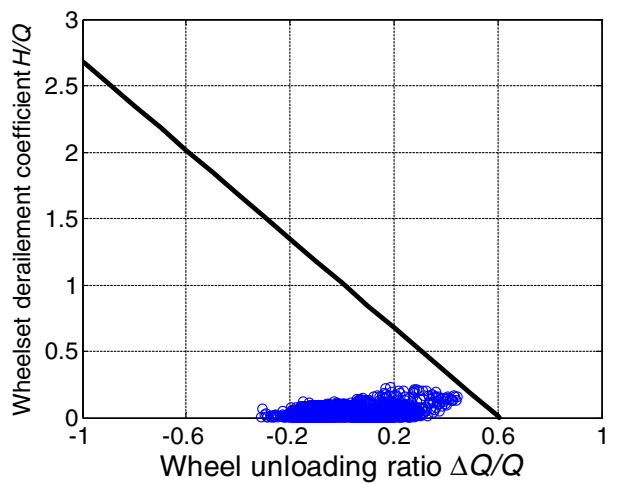

(b)

Fig. 5 Safety assessment against derailment for high-speed train under cross winds. a Head car. b Tail car 


\subsection{Identification of high-frequency wheel-rail forces}

The working mechanism of the instrumented wheelset is to measure the deformation of the wheel hub or axle. However, the deformation of the wheelset in high frequencies (e.g., caused by rail defects and wheel out-of-roundness) may come from the shift of wheel-rail contact points or the elastic deformation itself. In addition, the measurement of high-frequency force puts forward higher requirements on the signal transition system. Therefore, it is necessary to discuss the feasibility of applying this indirect method to measure the high-frequency forces. Under the interaction of short-wavelength track excitations, the acceleration generated on the wheelset is very high, while the dis- placement of the primary spring is very small and can be ignorable. Thus, Eqs. (1)-(3) can be further written as

$H=-m_{\mathrm{w}} a_{y}$,

$Q_{1}=G / 2+m_{\mathrm{w}} a_{z} / 2+H \cdot r_{0} / l_{\mathrm{c}}$,

and

$Q_{2}=G / 2+m_{\mathrm{w}} a_{z} / 2-H \cdot r_{0} / l_{\mathrm{c}}$.

It should be noted that this method is only applicable to the rigid body system in which the elastic vibration of the wheelset is not considered. To verify the indirect method theoretically, the wheel-rail forces determined from Eqs. (10)-(12) are compared with those calculated by Simpack. The running speed for the simulations is $50 \mathrm{~km} / \mathrm{h}$ for the wheel in flat condition with a wavelength of $30 \mathrm{~mm}$.

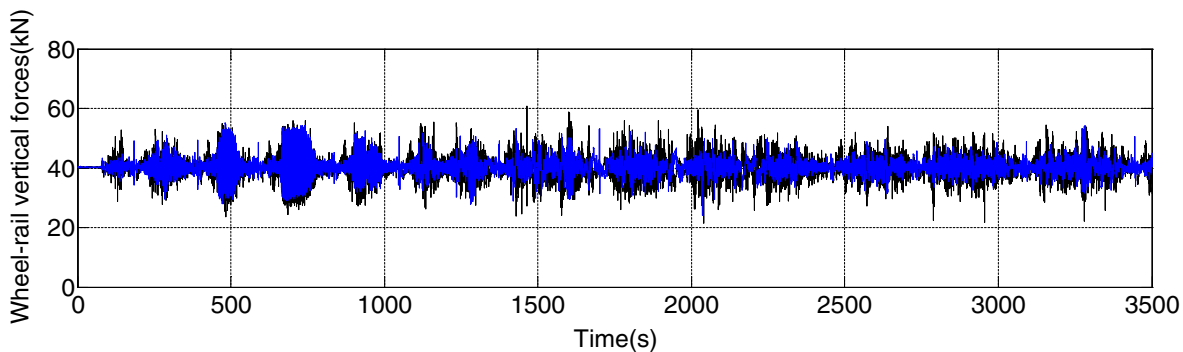

(a)

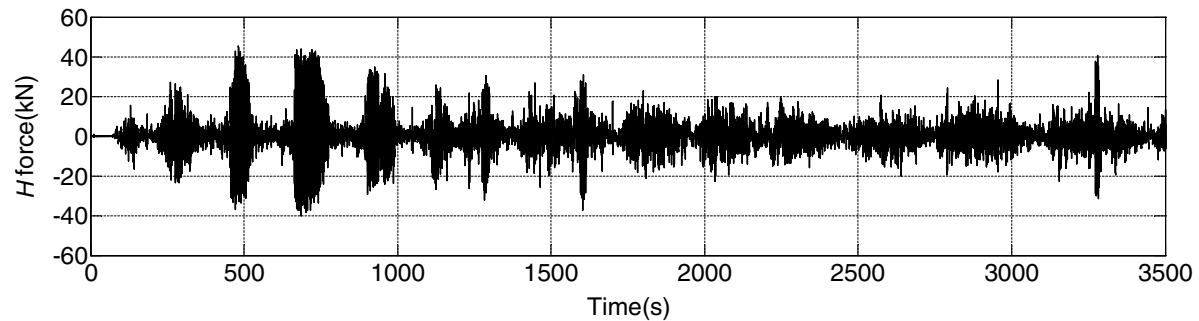

(b)

Fig. 7 Measured wheel-rail interaction forces of the metro vehicle with hunting motions. a Wheel-rail vertical forces. b $H$ force

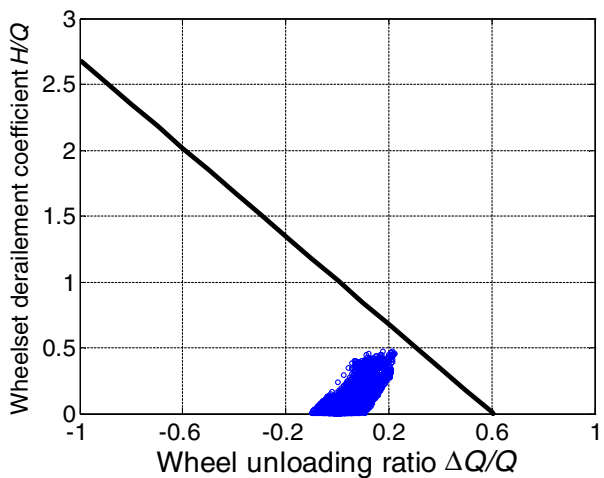

(a)

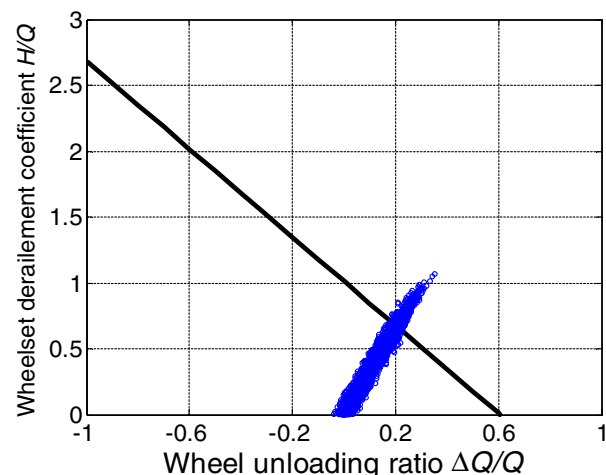

(b)

Fig. 8 Safetyassessment against derailment for metro vehicle with hunting motions. a Under stable condition. b Under unstable condition 

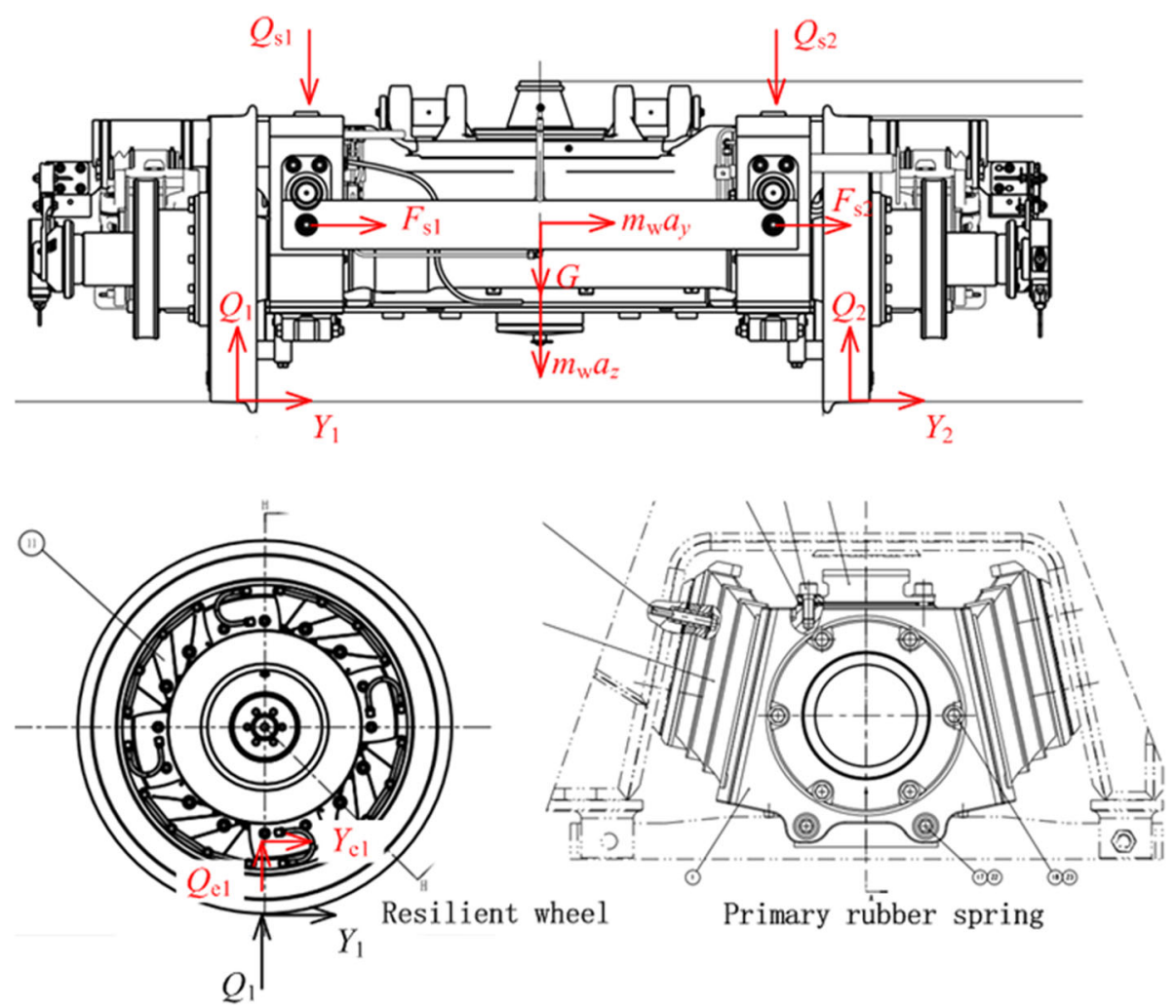

Primary rüber spring

Fig. 9 Analysis of forces exerted on resilient wheelset for LRV

The comparison of the identified wheel-rail vertical force with the output value of Simpack is shown in Fig. 11, from which we can see that this method gives good results in the time history.

In fact, the accelerations on the axle box due to elastic vibrations are apparently higher than that due to rigid motions. In future applications, the measured accelerations are necessary to be processed with a band pass filter so as to eliminate the influence of high-frequency elastic vibrations. The cutoff frequency of the filter will be determined by the characteristic frequency due to the wheel out-ofroundness or rail corrugation. For example, the characteristic frequency due to rail corrugation can be calculated by the wavelength and train speed.

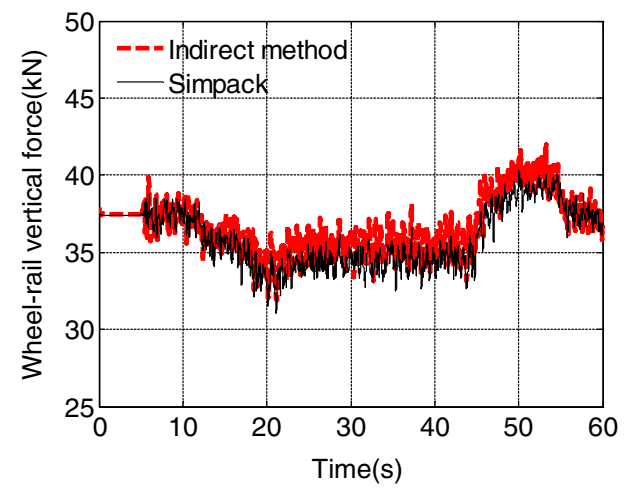

(a)

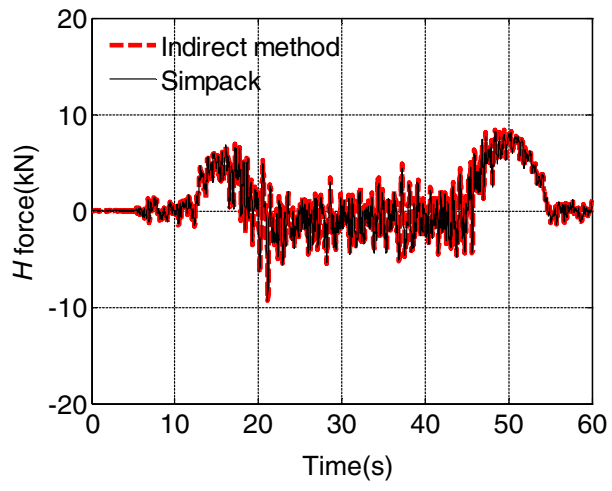

(b)

Fig. 10 Theoretical verifications by simulations on a curved track with radius of $300 \mathrm{~m}$. a Wheel-rail vertical forces. b $H$ force 


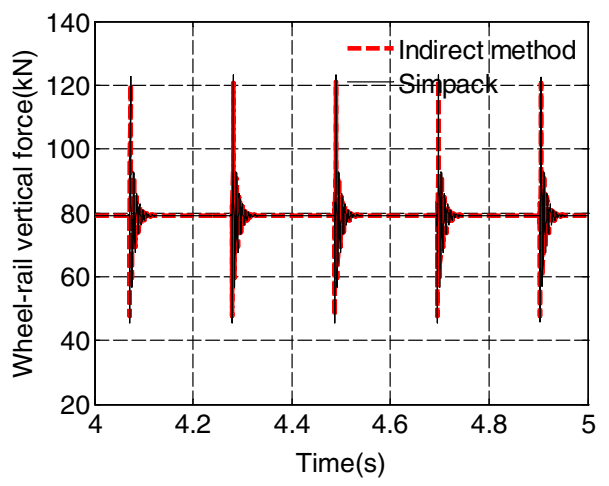

(a)

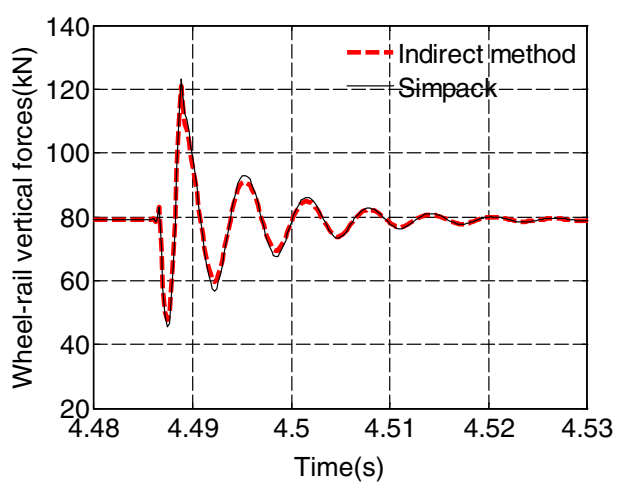

(b)

Fig. 11 Theoretical verifications by simulations for the wheel in flat condition. a Wheel-rail vertical forces. b Partial enlargement of one waveform

\section{Conclusions}

An improved indirect method for wheel-rail force measurement is proposed to evaluate the derailment safety of railway vehicles in various scenarios. From the applications, the following conclusions can be drawn:

(1) A union safety domain against derailment based on the wheelset derailment coefficient and the wheel unloading ratio is presented. The derailment safety can be evaluated by judging whether the scatter points are within the boundary limit of the triangular safety domain.

(2) Aiming at the high-speed train subjected to cross winds, the dynamic test using indirect method for wheel-rail force measurement was conducted. The results show that the wheel facing to the wind is loaded while the wheel away from the wind unloaded. The combination of the increasing lateral force and decreasing vertical force could result in derailment risk. The safety indices of the head car subjected to cross winds exceed the allowable limit in some locations, while the measured indices for the tail car under cross winds are within the safety limit.

(3) The dynamic test using the indirect method for wheel-rail force measurement for a metro vehicle was also conducted. It is found that the $H$ force increases significantly and hunting instability occurs in certain sections of the track. Similarly, the safety indices for the metro vehicle with hunting motions exceed the allowable limit, while that in the stable conditions, are within the safety limit.

(4) The forces exerted on a resilient wheelset of a light rail vehicle are analyzed. To verify the indirect method theoretically, the identified wheel-rail forces are compared with those calculated by Simpack. The results show that the $H$ force and the wheel-rail vertical forces identified by the proposed indirect method agree well with the calculated values by Simpack.

(5) The feasibility of applying this indirect method to measure the high-frequency forces is also discussed. The identified wheel-rail vertical force is compared with the output value by Simpack, showing a good coincidence in the time history of wheel-rail vertical force. However, this method is only applicable to the rigid body system in which the elastic vibration of the wheelset is not considered. Therefore, the measured accelerations are necessary to be processed with a band pass filter so as to eliminate the influence of high-frequency elastic vibrations.

Acknowledgments This work was supported by the National Natural Science Foundation of China (Grant No. U1334206 and No. 51475388) and Science \& Technology Development Project of China Railway Corporation (Grant No. J012-C).

Open Access This article is distributed under the terms of the Creative Commons Attribution 4.0 International License (http:// creativecommons.org/licenses/by/4.0/), which permits unrestricted use, distribution, and reproduction in any medium, provided you give appropriate credit to the original author(s) and the source, provide a link to the Creative Commons license, and indicate if changes were made.

\section{References}

1. Riggall G (2008) IWT4 goes into operation. Railw Gazette Int 164:455-456

2. Braghin F, Bruni S, Cervello S, Cigada A, Resta F (2003) A new method for the measure of the wheel-rail contact forces. In: Proceedings of the 6th international conference on contact mechanics and wear of rail/wheel systems (CM2003), Goteborg, 313-319 
3. Matsumoto A, Sato Y, Ohno H, Shimizu M, Kurihara J et al (2012) Continuous observation of wheel/rail contact forces in curved track and theoretical considerations. Veh Syst Dyn 50(sup1):349-364

4. Kanehara H, Fujioka $T$ (2002) Measuring rail/wheel contact points of running railway vehicles. Wear 253(1):275-283

5. Ham YS, Lee DH, Kwon SJ, You WH, Oh TY (2009) Continuous measurement of interaction forces between wheel and rail. Int $\mathbf{J}$ Precis Eng Manuf 10(1):35-39

6. Elkins JA, Carter A (1993) Testing and analysis techniques for safety assessment of rail vehicles: the state-of-the-art. Veh Syst Dyn 22(3-4):185-208

7. Papini S, Pugi L, Rindi A, Meli E (2013) An integrated approach for the optimization of wheel-rail contact force measurement systems. J Mod Transp 21(2):95-102

8. GB5599-85 (1985) Railway vehicles-specification for evaluation the dynamic performance and accreditation test, National Bureau of Standard, Beijing (in Chinese)

9. Genaro G, Rade DA (1998). Input force identification in the time domain. In: SPIE proceedings series, Society of Photo-Optical Instrumentation Engineers, pp. 124-129

10. Parloo E, Verboven P, Guillaume P, Van Overmeire M (2003) Force identification by means of in-operation modal models. J Sound Vib 262(1):161-173

11. Xia F, Cole C, Wolfs P (2007) An inverse railway wagon model and its applications. Veh Syst Dyn 45(6):583-605

12. Xia F, Cole C, Wolfs $P$ (2008) Grey box-based inverse wagon model to predict wheel-rail contact forces from measured wagon body responses. Veh Syst Dyn 46(S1):469-479

13. Uhl T (2007) The inverse identification problem and its technical application. Arch Appl Mech 77(5):325-337
14. Zhu T, Xiao S, Yang G, Ma W, Zhang Z (2014) An inverse dynamics method for railway vehicle systems. Transport 29(1):107-114

15. Mehrpouya M, Ahmadian H (2009) Estimation of applied forces on railway vehicle wheelsets from measured vehicle responses. Int J Veh Struct Syst 1(4):104-110

16. Tanaka H, Furukawa A (2008) The estimation method of wheel load and lateral force using the axlebox acceleration. WCRR, Seoul

17. Gao C, Lu H, Ren L (2012) Test on the wheel/rail forces of $70 \%$ low-floor tram. Urban mass transit 15(10):43-45 (in Chinese)

18. Wang W, Wang Y, Sun S, Liang S (2015) Long-term load spectrum test of high speed train bogie. J Southwest Jiaotong Univ 50(1):84-89 (in Chinese)

19. Matsumoto A, Sato Y, Ohno H, Tomeoka M, Matsumoto K, Kurihara K, Nakai T (2008) A new measuring method of wheelrail contact forces and related considerations. Wear 265(9):1518-1525

20. Wei L, Zeng J, Wu P, Gao H (2014) Indirect method for wheelrail force measurement and derailment evaluation. Veh Syst Dyn 52(12):1622-1641

21. Wei L, Zeng J, Wu P, Gao H, Wang Q (2015) Derailment safety evaluation for railway vehicles based on wheelset model. J China Railw Soc 37(9):25-31 (in Chinese)

22. Wu P, Wei L, Zeng J, Gao H (2015) Safety assessment of high speed trains under cross winds. In: The 24th international symposium on dynamics of vehicles on roads and tacks (ISVSD 2015), Graz

23. Zeng J, Guan Q (2008) Study on flange climb derailment criteria of a railway wheelset. Veh Syst Dyn 46(3):239-251 